\title{
Comparative molecular analysis of ovine and bovine Streptococcus uberis isolates
}

\author{
T. L. Gilchrist, ${ }^{*}$ D. G. E. Smith, ${ }^{*} \dagger$ J. L. Fitzpatrick, ${ }^{*}$ R. N. Zadoks, ${ }^{*} \ddagger^{1}$ and M. C. Fontaine ${ }^{\star 1,2}$ \\ *Moredun Research Institute, Pentlands Science Park, Bush Loan, Penicuik EH26 OPZ, United Kingdom \\ †Institute of Infection, Inflammation and Immunity, College of Medicine, Veterinary Medicine and Life Sciences, University of Glasgow, \\ 464 Bearsden Road, Glasgow G61 1QH, United Kingdom \\ ‡Royal (Dick) School of Veterinary Studies, University of Edinburgh, Roslin EH25 9RG, United Kingdom
}

\begin{abstract}
Streptococcus uberis causes clinical and subclinical mastitis in cattle and sheep, but it is unknown whether the composition of Strep. uberis populations differs between host species. To address this, we characterized a collection of bovine and ovine Strep. uberis isolates with shared geographical and temporal origins by means of an expanded multilocus sequence typing scheme. Among 14 ovine and 35 bovine isolates, 35 allelic profiles were detected. Each allelic profile was associated with a single host species and all but one were new to the multilocus sequence typing database. The median number of new alleles per isolate was higher for ovine isolates than for bovine isolates. None of the ovine isolates belonged to the global clonal complexes 5 or 143, which are commonly associated with bovine mastitis and which have a wide geographical distribution. Ovine isolates also differed from bovine isolates in carriage of plasminogen activator genes, with significantly higher prevalence of $p a u B$ in ovine isolates. Isolates that were negative for yqiL, one of the targets of multilocus sequence typing, were found among ovine and bovine isolates and were not associated with a specific sequence type or global clonal complex. One bovine isolate carried a gapC allele that was probably acquired through lateral gene transfer, most likely from Streptococcus salivarius. We conclude that ovine isolates are distinct from bovine isolates of Strep. uberis, and that recombination between isolates from different host species or bacterial species could contribute to changes in virulence gene profiles with relevance for vaccine development.
\end{abstract}

Key words: Streptococcus uberis, molecular typing, ovine, bovine

Received May 9, 2012.

Accepted October 17, 2012.

${ }^{1}$ Contibuted equally to this research.

${ }^{2}$ Corresponding author: michael.fontaine@moredun.ac.uk

\section{INTRODUCTION}

Streptococcus uberis is an important mastitis-causing pathogen in dairy cattle in many parts of the world, including Australia and New Zealand (McDougall et al., 2004; Shum et al., 2009), North America (Rossitto et al., 2002; Olde Riekerink et al., 2008), and Europe (Bradley et al., 2007; Sampimon et al., 2009). Multilocus sequence typing (MLST) has been used to explore the population structure of Strep. uberis from different countries and to identify groups of genetically related sequence types $(\mathbf{S T})$, called clonal complexes $(\mathbf{C C})$ or, if they are very large, global clonal complexes (GCC; Pullinger et al., 2006; Rato et al., 2008; Tomita et al., 2008). In addition, the relationship between ST or CC and sources or clinical manifestations of Strep. uberis mastitis has been investigated (López-Benavides et al., 2007; Pullinger et al., 2007; Tomita et al., 2008). In the United Kingdom, GCC5 is the most common group of Strep. uberis strains isolated from milk, whereas in New Zealand, GCC143 is the most common group, but members of both GCC occur in multiple countries (Pullinger et al., 2006). Global clonal complexes 5, 86, and 143 have been associated with clinical mastitis, latent infection, and subclinical mastitis, respectively (Tomita et al., 2008), but there does not appear to be an association between ST or CC and duration of infection (Pullinger et al., 2007). In addition to the dominant $\mathrm{CC}$, a large number of singletons exist; that is, ST that differ from all other ST at more than one locus. The high level of heterogeneity in the Strep. uberis population is partly attributed to its recombinatorial population structure (Zadoks et al., 2005; Coffey et al., 2006; Lang et al., 2009).

Streptococcus uberis may also cause clinical or subclinical mastitis in sheep (Zadoks, 2007). In some studies from the United States and the United Kingdom, detection of Strep. uberis in ovine milk samples was rare and not associated with an increase in SCC (Kirk et al., 1980; Hariharan et al., 2004). In a study from Turkey, however, Strep. uberis was detected in $7 \%$ of udder halves from mastitic sheep, making it the third 
most common pathogen isolated from sheep udders at slaughter (Gülcü and Öngör, 2002). It was also detected in $10 \%$ of 1,186 culture-positive samples from sheep in Italy, and detection of streptococci was associated with a significant increase in SCC compared with detection of staphylococci (Cuccuru et al., 2011). Whole-genome and MLST studies of Staphylococcus aureus from cattle and sheep have led to identification of strains that are specifically associated with one host species (Ben Zakour et al., 2008; Vautor et al., 2009). In contrast, molecular analyses of Strep. uberis isolates associated with ovine mastitis have not been conducted, and there is a lack of knowledge regarding the relationship between Strep. uberis from ovine and bovine populations. To gain insight into the population structure of ovine Strep. uberis, we conducted comparative molecular analysis of a collection of Strep. uberis field isolates from cases of ovine and bovine mastitis with a shared geographical and temporal origin. In addition to MLST based on housekeeping genes (Coffey et al., 2006), we used analysis of the genes encoding plasminogen activator A (PauA) and glyceraldehyde3-phospate dehydrogenase (GapC). These targets have been associated with Strep. uberis virulence as well as efforts to develop mastitis vaccines (Leigh et al., 1999; Fontaine et al., 2002), and therefore may have specific clinical relevance.

\section{MATERIALS AND METHODS}

\section{Bacterial Isolates and DNA Preparation}

Streptococcus uberis isolates for MLST were kindly provided in pure culture by Francesco Cancellotti (Istituto Zooprofilattico Sperimentale delle Regioni Lazio e Toscana, Rome, Italy), and comprised 49 isolates from cases of ovine $(\mathrm{n}=14)$ and bovine $(\mathrm{n}=35)$ mastitis (clinical or subclinical) collected in 2006 or 2007 in the Lazio province of Italy. Upon receipt, strains were grown in brain heart infusion broth (Oxoid, Basingstoke, UK) and cultivated statically at $37^{\circ} \mathrm{C}$ for 20 to $24 \mathrm{~h}$. Culture purity and colony morphology was examined on blood agar plates, prepared with blood agar base (Oxoid) supplemented with $5 \%$ sheep blood. Species identity was confirmed using the API 20 Strep kit (bioMérieux, Marcy l'Etoile, France) according to the manufacturer's guidelines, and species-specific $16 \mathrm{~S}$ rRNA PCR (Hassan et al., 2001).

Genomic DNA was extracted using the NucleoSpin Tissue Kit (Machery Nagel, Düren, Germany) following the manufacturer's guidelines, with minor modifications: Strep. uberis was cultured in $2 \mathrm{~mL}$ of brain heart infusion broth for 20 to $24 \mathrm{~h}$ to obtain stationary-phase cultures. Cultures were centrifuged for $15 \mathrm{~min}$ at 2,000 $\times g$, and cell pellets were resuspended in $300 \mu \mathrm{L}$ of gram-positive lysis buffer $(20 \mathrm{~m} M$ Tris- $\mathrm{HCl}, \mathrm{pH} 8.0$, $2 \mathrm{mM}$ EDTA, $1 \%$ Triton X 100, and $2 \mathrm{mg} / \mathrm{mL}$ lysozyme). Before addition of $2.5 \mathrm{mg} / \mathrm{mL}$ proteinase $\mathrm{K}$, $100 \mu \mathrm{L}$ of lysate was discarded to avoid overloading the NucleoSpin columns. Genomic DNA was eluted in $60 \mu \mathrm{L}$ of prewarmed buffer BE containing $20 \mu \mathrm{g} / \mathrm{mL}$ RNaseA, quantified using a NanoDrop ND-100 UV-Vis spectrophotometer (NanoDrop Technologies, Wilmington, DE), and analyzed by agarose gel electrophoresis. Samples were diluted to $100 \mathrm{ng} / \mu \mathrm{L}$ for use in PCR.

\section{PCR and DNA Sequencing}

A 7-gene MLST panel (Coffey et al., 2006) was used to determine the population structure of the isolates included in this study. Amplification of the $t d k$ region using the $t d k$ primers described by Coffey and coworkers (2006) failed to identify a single, clear PCR product. The published Strep. uberis 0140J genome sequence (GenBank accession number NC_012004; http://www. ncbi.nlm.nih.gov/nuccore/NC_012004.1; Ward et al., 2009) was therefore used as a template for designing new $t d k$-specific primers (Table 1). Analysis of 2 additional genes (pauA and gapC) was conducted using PCR primers described as part of an alternative MLST scheme (Zadoks et al., 2005). Where PCR failed to amplify a product from pauA using the primers described by Zadoks and colleagues (2005), alternative primers (ER45 and ER46), which included the pauA intergenic region, were used. Sequencing of the PCR product derived from pauA-negative isolates using the ER45 and ER46 primers permitted the design of additional primers ( pauB F and $\mathrm{R}$ ) to sequence a larger region of the gene. Details of primers used and conditions required for amplification are listed in Table 1.

The PCR products were purified using the MinElute 96 UF PCR Purification kit (Qiagen, Sussex, UK) according to the manufacturer's protocol and eluted in 30 $\mu \mathrm{L}$ of sterile molecular biology-grade water. Sequencing was conducted in both directions by commercial companies, using the same primers as used for PCR.

\section{Molecular and Data Analysis}

Forward and reverse sequences were aligned using Clone Manager Professional Suite v. 9 (Scientific and Educational Software, Cary, NC) and the sequence files converted to FASTA format. Using SeqMan (DNAStar Inc., Madison, WI), sequences were trimmed to correspond with alleles from the Strep. uberis PubMLST database (http://pubmlst.org/suberis/), and sequences were compared against all existing allelic types from the database. When no match to previous entries was 
Table 1. Oligonucleotide primer sequences and amplification conditions used for PCR and sequencing

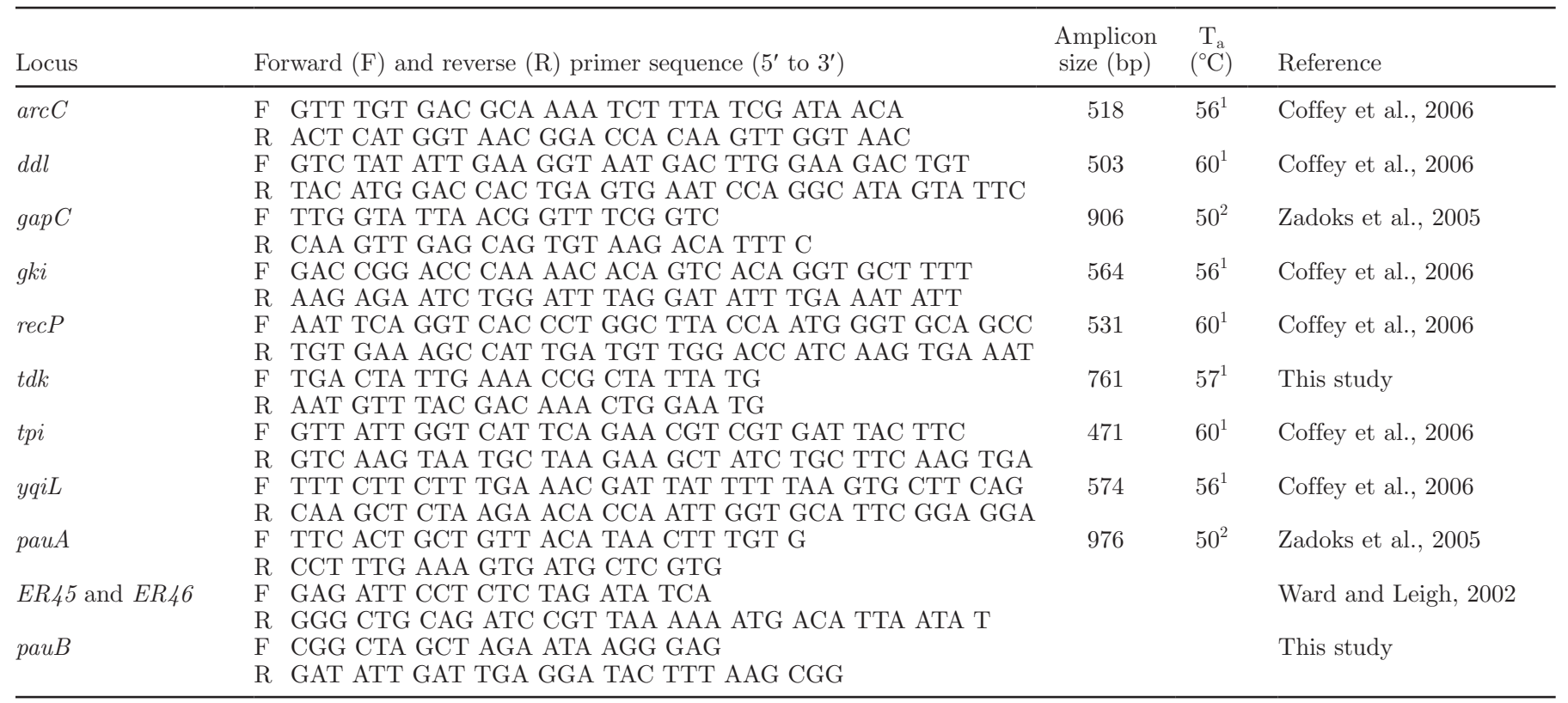

${ }^{1} \mathrm{PCR}$ conditions were $5 \mathrm{~min}$ at $94^{\circ} \mathrm{C}, 40$ cycles of $\left[30 \mathrm{~s}\right.$ at $94^{\circ} \mathrm{C}, 30 \mathrm{~s}$ at annealing temperature, $\mathrm{T}_{\mathrm{a}}$, and $45 \mathrm{~s}$ at $\left.72^{\circ} \mathrm{C}\right]$, and $7 \mathrm{~min}$ at $72^{\circ} \mathrm{C}$.

${ }^{2} \mathrm{PCR}$ conditions were $5 \mathrm{~min}$ at $94^{\circ} \mathrm{C}, 40$ cycles of $\left[30 \mathrm{~s}\right.$ at $94^{\circ} \mathrm{C}, 60 \mathrm{~s}$ at $\mathrm{T}_{\mathrm{a}}$, and $45 \mathrm{~s}$ at $\left.72^{\circ} \mathrm{C}\right]$, and $7 \mathrm{~min}$ at $72^{\circ} \mathrm{C}$.

found, the original trace files were sent to the database curator for assignment of new alleles. Allelic profiles (AP) were obtained and compared with the PubMLST database; when these profiles were novel, they were assigned new ST by the database curator if sequence data were available for 7 alleles. Allele numbers for gap $C$ and pauA were assigned in accordance with the in-house database for those genes.

Associations between binary variables; for example, host species (ovine vs. bovine) and presence or absence of specific PCR products, were explored using $2 \times 2$ tables and the Fisher exact test. Associations between binary variables, such as host species, and nonbinary variables, such as number of new alleles, were tested using the Wilcoxon rank sum test. Analyses were conducted with 3 sets of input data: (1) at isolate level (n $=49$ ); (2) at nonduplicate level (i.e., if multiple isolates shared the same AP and origin, this combination was only included once but if multiple isolates with the same AP came from different origins, one representative from each origin was included; $\mathrm{n}=40$ ); (3) at AP level $(\mathrm{n}=35)$. Statistical analyses were conducted using Statistix 9.0 (Analytical Software, Tallahassee, FL). Because of the limited number of ovine isolates, statistical significance was declared at $P<0.10$.

Relationships between ST from the current study and the PubMLST database were explored using eBURST V3 (http://eburst.mlst.net/), with grouping of ST that shared at least 6 of 7 alleles. The origin of isolates that shared ST or eBURST groups with isolates from the current study was obtained from the isolate database for Strep. uberis (http://pubmlst.org/suberis/; database last updated April 15, 2010, last accessed January 4, 2012).

\section{RESULTS}

\section{Host Association of Allelic Profiles}

Among 14 ovine and 35 bovine isolates, 35 AP were detected. Most AP were represented by a single isolate. Of 9 AP that were represented by multiple isolates, 5 originated from a single region and 4 originated from multiple regions (Table 2). Each of the 5 AP that originated from a single region was included once in statistical analyses at the nonduplicate level, whereas AP that originated from multiple regions were included twice or 3 times, depending on the number of regions of origin. Each AP was associated with a single host species (Table 2). With the exception of one isolate, all isolates belonged to AP that were new to the Strep. uberis MLST database (Table 2). Of 34 new AP, 10 were the result of new combinations of existing alleles, and $24 \mathrm{AP}$ included alleles that were new to the database. The number of new alleles per ST ranged from 1 to 4 , with a median of 2 , and was higher for ovine than for bovine isolates (Wilcoxon rank sum test; $P<0.10$ at isolate, nonduplicate, and AP levels). 
Table 2. Allelic profiles of ovine and bovine Streptococcus uberis isolates from this study ${ }^{1}$

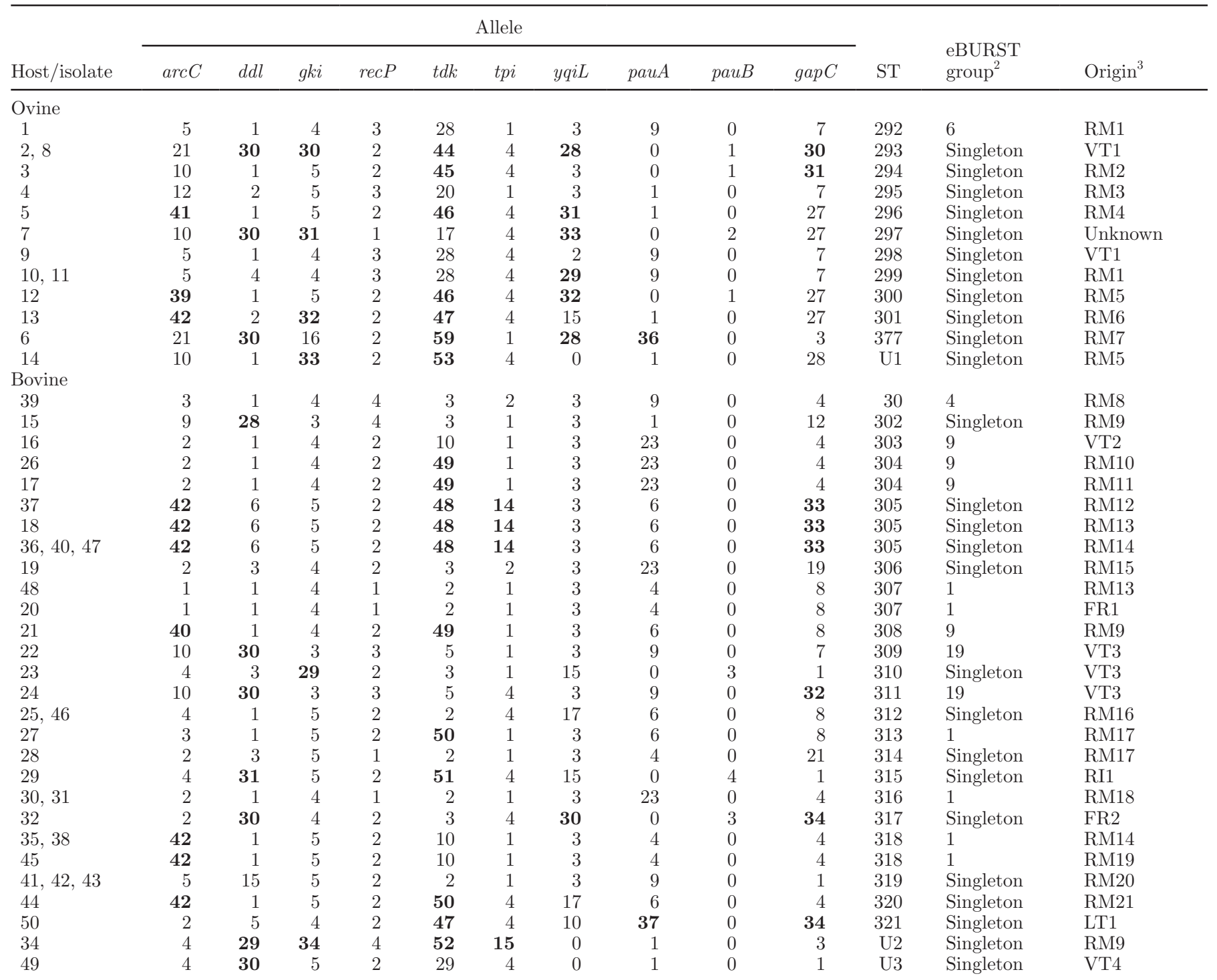

${ }^{1}$ New alleles are shown in bold face. Sequence types (ST) were assigned to isolates that had completed allelic profiles for $\operatorname{arc} C, d d l, g k i, r e c P, t d k$, tpi, and yqiL and are otherwise listed as unknown (U). A value of zero indicates absence of PCR product for the target gene.

${ }^{2}$ Based on eBURST (http://eburst.mlst.net/) analysis using similarity at 6 of 7 loci as grouping criterion, and using PubMLST profiles database (http://pubmlst.org/suberis/) from July 20, 2011. eBURST groups may change in size, composition, and number assigned to the group as the database evolves.

${ }^{3}$ Letters indicate province in the Lazio region of Italy: FR $=$ Frosinone, LT = Latina, RI $=$ Rieti, $\mathrm{RM}=\mathrm{Roma}$, and VT $=\mathrm{Viterbo}$; numbers indicate individual farms or veterinary practices.

\section{Host Association of Plasminogen Activator Alleles}

Isolates that belonged to the same $\mathrm{AP}$ also shared the same pauA or pauB and gapC alleles (Table 2). Allele pauB could be amplified from all isolates that failed to yield a pauA PCR product $(\mathrm{n}=7)$. Presence of pauB was more common among ovine isolates than among bovine isolates. The difference was significant at the isolate level, but not at the nonduplicate or AP levels (Wilcoxon rank sum test; $P=0.03, P=0.10$, and $P=0.16$, respectively). Four pauB alleles were detected (Figure 1); pauB allele 1 was only detected in sheep, but it was associated with multiple AP and geographic origins, whereas pauB allele 3 was only detected in cattle, and was associated with multiple AP and geographic origins (Table 2). Isolates with pauB had significantly more new alleles than isolates with pauA (Wilcoxon rank sum test; $P<0.03$ at the iso- 
late, nonduplicate, and AP levels). All pauA-negative isolates differed at 4 or more alleles from previously published pauA-negative isolates (Lang et al., 2009).

\section{Isolates Negative for yqiL}

Of 49 isolates, 3 were negative for yqiL; the yqiLnegative isolates included 1 ovine and 2 bovine isolates, which belonged to 3 unrelated AP and came from 3 different geographic origins. All yqiL-negative isolates were positive for pauA (Table 2). In the online database, ST are not assigned to isolates that are yqiLnegative, hence the use of AP rather than ST to allow for description of all isolates in the current study. The yqiL-negative isolates differed from all ST in the online database in 3 or more alleles, counting absence versus presence of yqiL as an allelic difference. All yqiLnegative isolates from the current study differed from previously described yqiL-negative isolates in at least 4 alleles (Tomita et al., 2008; Table 3).

\section{Host Association and Geographic Origin of eBURST Groups}

One ovine isolate and 15 bovine isolates belonged to ST that were part of an eBURST group; that is, a group of ST that were single locus variants (SLV) of each other. The remaining isolates belonged to singletons (Table 2). Ovine isolates were significantly more likely than bovine isolates to belong to singletons (Fisher Exact; $P<0.05$ at the isolate, nonduplicate, and ST levels). The most common eBURST group was group 1, which includes GCC5 and GCC143. Eight bovine isolates belonged to this group, but none of the ovine isolates did (Table 2). The difference was significant (Fisher Exact; $P<0.10$ ) at the isolate and nonduplicate levels, but not at the ST level.

The only ovine isolate that was part of an eBURST group belonged to ST292 and eBURST group 6 (Figure $2)$. The members of this group originate from Sweden, Italy, and the UK. Sequence type 292 is the only member of the group to have been isolated from ovine rather than bovine milk. One bovine isolate belonged to ST30, which is at the core of eBURST group 4. This group includes multiple ST from multiple countries (Figure 2). Two bovine isolates belonged to eBURST group 9, which consists of $2 \mathrm{ST}$ from the current study that are SLV of each other; and 2 isolates belonged to eBURST group 19, which also consists of 2 ST from the current study that are SLV of each other. Three bovine ST from the current study-ST303, ST318, and ST319-had previously been identified in Italy (www.pubmlst.net/ suberis). Isolates with these ST originated from 1, 3, and 1 Italian dairy herd(s), respectively, and all herds were unrelated to the flocks and herds involved in the current study (Paolo Moroni, Università degli Studi di Milano, Milan, Italy; personal communication).

\section{Diversity of Glyceraldehyde-3-Phospate Dehydrogenase}

The majority of $g a p C$ alleles from Italian isolates were previously described or diverged from existing gap C alleles by 0.1 to $0.9 \%$ (sequence heterogeneity). One bovine isolate (isolate 24; Table 2) had a gapC allele that diverged from all other alleles by 15.3 to $16.1 \%$, which is more than the average difference $(10 \%)$ between the gapC allele from Streptococcus parauberis (Zadoks et al., 2005) and gapC alleles of Strep. uberis. BLAST analysis of the divergent gapC allele revealed $99.9 \%$ identity (840 of 841 nucleotides) with the gapC allele of Streptococcus salivarius (CP00288.1 and FR873481.1), whereas the next best matches showed $91 \%$ or less identity. The isolate with the highly divergent $\operatorname{gap} C$ allele was an SLV of another isolate in the current study.

\section{DISCUSSION}

To our knowledge, there has been no previous investigation of Strep. uberis isolated from cases of ovine mastitis. Despite the observed heterogeneity of the bovine-derived Strep. uberis population, the ovine isolates described in this study were recognizably distinct from bovine isolates obtained from the same region during the same sampling period. Ovine isolates contained a larger median number of new alleles per AP, were more likely to belong to singletons, and less likely to be related to GCC5 and GCC143 than bovine isolates. Taken together, these data would suggest that different subpopulations of Strep. uberis might be better adapted to cause mastitis in cattle or sheep. Although gastrointestinal carriage of Strep. uberis in cattle has been described, no such data are available for sheep (Zadoks, 2007). Consequently, it is not possible to state whether differences between ovine and bovine mastitis-causing strains result from initial adaptation to a primary niche (i.e., the gastrointestinal tract), a subsequent adaptation to the mammary gland environment, or both. The existence of apparently distinct Strep. uberis populations in cows and sheep suggests that mastitis arises as a result of infection from the host's resident population rather than from environmental sources that are common to both host species.

Considering the heterogeneity of the global Strep. uberis population, any MLST-based study of Strep. uberis is likely to yield new ST. Some ST are overrepresented in specific geographic areas; for example, 


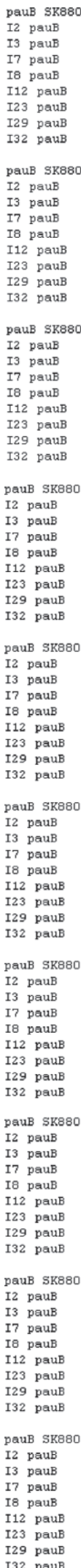

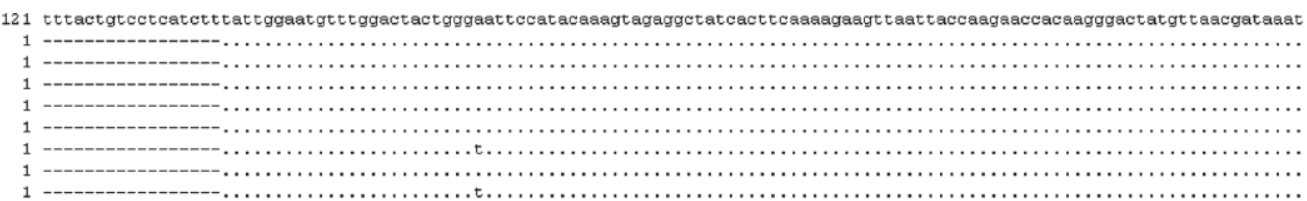

241 attaccggagaaagcaaggagggacagttgetcetttetccagaatatatgcaatttegettaacagcgggagaagt tat taataaaaatgagttactggataaagtgeaact tgttat

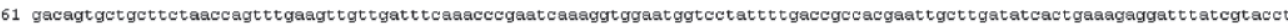
224

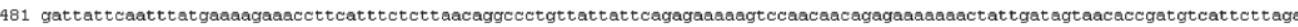

601 cacagcgtttcetttatgaaaagaaacggegaatcaatgaatettgttccaacaactacacagetatcttcattttctaaaact gcaaaacctggtcagtcaattcaatcagaagaactc 464

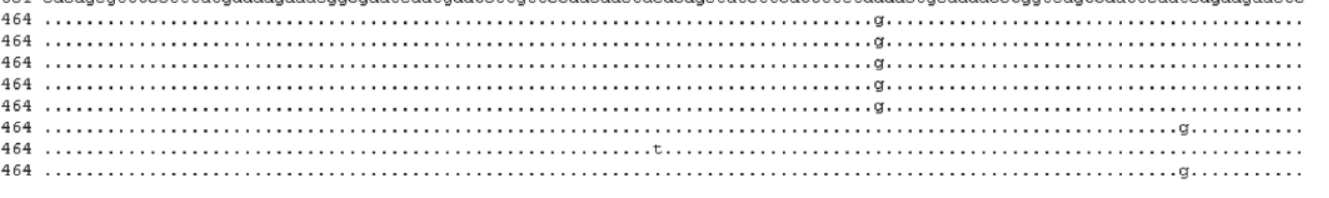

721 tatcaagctgctcaatcact ttttgagcaaacaaagaatatcatgaaggt tatcaattaatcaaacggt tgaacacatcgat $t$ cagaaaatgaaaaagcctatcgcagtgt $t$ atcae

584

584

584

584

841 tttcaaaacggaaaccettcaactatacgat teacaactaagagagttttcaaaatgggat tat tcacaa----------------gcaattagagacgatatcat tgagaactat tac

704

704

704
704
704

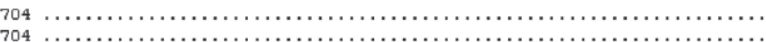

946 att 809

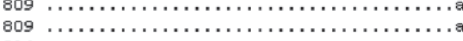
824 . 809 .

809

809

1066 gagcatat taaacactatct tagtgaagcagatctcactgaaat tgatgetaaaacaggagatcattattggtttagtcagaatgtcaagcaggtatcatcecaccatttccaggetaac 929 .

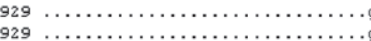

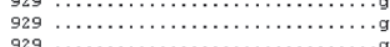

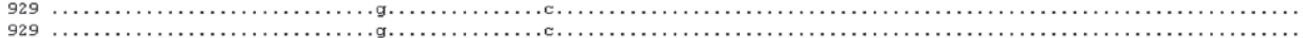

1186 tatt cagcaaagaaaactctgatt gttetactataacegattcagettgeaataattggtactgaaataaagtgacgaagact aacaggtettcgttttttgettctcatttttgat 1049 1049

1064

1049

1049

1049

Figure 1. Alignment of novel pauB sequences from Streptococcus uberis with reference sequence AJ314852. Isolates I2, I3, I7, I8, and I12 originate from ovine milk; isolates I23, I29, and I32 originate from bovine milk. Nucleotide polymorphisms are highlighted. 
Table 3. Comparison of allelic profiles for ovine and bovine yqiL-negative isolates of Streptococcus uberis

\begin{tabular}{lcrrrrrll}
\hline & \multicolumn{9}{c}{ Allele } & & \\
\cline { 2 - 6 } Host species & arcC & $d d l$ & gki & recP & $t d k$ & $t p i$ & Country & Reference \\
\hline Ovine & 10 & 1 & 33 & 2 & 53 & 4 & Italy & This study \\
Bovine & 4 & 29 & 34 & 4 & 52 & 15 & Italy & This study \\
Bovine & 4 & 30 & 5 & 2 & 29 & 4 & Italy & This study \\
Bovine & 1 & 1 & 3 & 2 & 1 & 1 & Australia & Tomita et al., 2008 \\
Bovine & 3 & 2 & 28 & 2 & 6 & 4 & Australia & Tomita et al., 2008 \\
Bovine & 6 & 14 & 4 & 15 & 22 & 13 & Australia & Tomita et al., 2008 \\
Bovine & 21 & 21 & 18 & 11 & 41 & 6 & Australia & Tomita et al., 2008 \\
\hline
\end{tabular}

members of GCC5 in the UK and members of GCC143 in New Zealand. These associations, however, are not absolute because members of GCC5, GCC86, and GCC143 have been observed in the UK, Portugal, Australia, and New Zealand (Rato et al., 2008; Zadoks et al., 2011). New ST in the current study, which is the first to describe Strep. uberis isolates from Italy, also included members from GCC5, GCC86, and GCC143. In addition, several ST, including the newly identified ST292, were members of smaller CC that included isolates from several European countries, including the UK, continental Europe, and southern as well as northern Europe. Apparent geographic clustering of ST may be a function of sample size and bacterial population heterogeneity rather than an indication of the existence of independently evolving populations. It will be interesting to see whether the apparent divergence of bovine and ovine isolates continues to be observed when larger collections of ovine isolates are identified. The fact that this divergence was observed among temporally matched isolates from a geographically limited area suggests that it may reflect a biological difference rather than being due to study design or sample size. Experimental studies using isolates of ovine and bovine origin to challenge cattle and sheep might provide further insight into potential biological differences.

Ovine Strep. uberis isolates differed from bovine isolates in the plasminogen activator-encoding gene that they carried; that is, they were more likely than bovine isolates to contain the alternative plasminogen activator pauB rather than the more common plasminogen activator pauA. Where pauB was present, alleles appeared to be host species-associated, although we recognize that the number of isolates per allele is currently too small to detect significant associations. Detection of pauB has been described previously in a bovine Strep. uberis isolate from Denmark (Ward and Leigh, 2002), and pauA negative isolates have been described in multiple studies (Khan et al., 2003; Zadoks et al., 2005). It is hypothesized that plasminogen activators contribute to the acquisition of essential nutrients from milk casein, increasing the ability of bacteria to survive in nu-

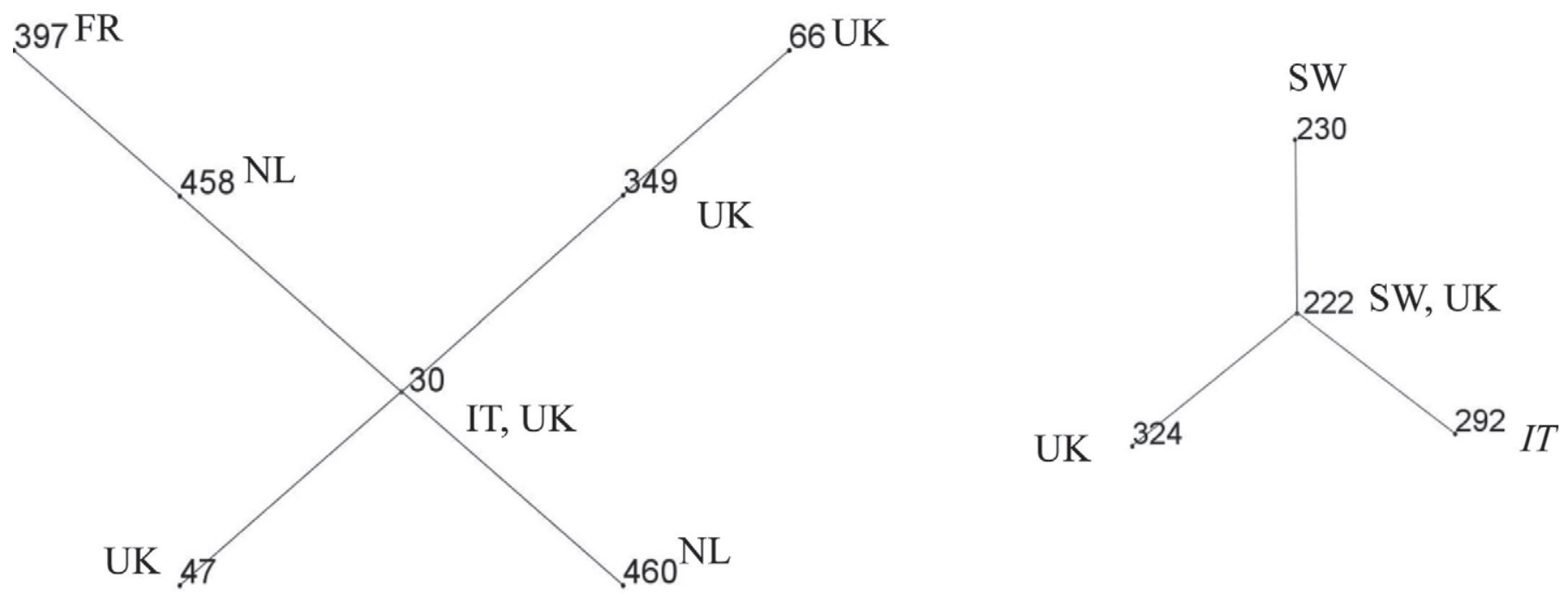

Figure 2. eBURST (http://eburst.mlst.net/) groups 4 (left) and 6 (right), as identified based on multilocus sequence typing of Streptococcus uberis, with country of origin: FR = France, IT = Italy, $\mathrm{NL}=$ the Netherlands, $\mathrm{SW}=$ Sweden, UK $=$ United Kingdom. Numbers indicate sequence types; text in italics indicates an isolate of ovine origin. 
tritionally limited environments (Kitt and Leigh, 1997) and to invade tissues via the breakdown of extracellular matrix components (Jones and Holt, 2004; Sun et al., 2004). PauA and PauB have equivalent capacities to activate bovine plasminogen but PauB has an enhanced capacity to activate ovine plasminogen compared with PauA (Ward and Leigh, 2002). The observed variation in carriage of genes such as pauA or pauB highlights a potential problem with respect to the development of vaccines against Strep. uberis, because vaccines based on a single strain or a single antigen may not provide protection against all strains of the pathogen. Furthermore, the highly recombinatorial nature of the Strep. uberis population and the potential ability for gene derivatives that predominate within a given environment (e.g., sheep) to cross into another environment (e.g., cattle) mean that vaccine design may need to take into account ovine-derived Strep. uberis strains, which thus far have not been considered.

The genetic heterogeneity of potential vaccine candidates is also illustrated by the presence of a $g a p C$ allele that diverged from known gapC alleles in Strep. uberis by more than $15 \%$, while showing $99.9 \%$ sequence identity to gapC of Strep. salivarius. Here, alleles from different bacterial species, rather than different host species, appear to contribute to this heterogeneity. Recently, the presence of the salivarin operon, which originates from Strep. salivarius, was described in several isolates of Streptococcus agalactiae derived from bovine mastitis (Richards et al., 2011), providing evidence that lateral gene transfer between Strep. salivarius and mastitis-causing streptococci is possible. Although Strep. salivarius is primarily an organism of the human oral cavity, the salivarin operon was not detected in human Streptococcus agalactiae isolates (Richards et al., 2011). It has been suggested that lateral gene transfer between Strep. salivarius and mastitis-causing streptococci may occur within the bovine environment (Richards et al., 2011), because Strep. salivarius has been isolated from bovine bulk tank milk and from quarter milk samples with SCC > 200,000 cells/mL (Zadoks et al., 2004; Nam et al., 2009).

The typeability of the isolates included in this study was $93.9 \%$ using the Coffey-MLST scheme (Coffey et al. (2006), owing to the presence of 3 yqiL-negative isolates. Similarly, 4 of 46 Australian Strep. uberis isolates failed to yield a yqiL amplicon (Tomita et al., 2008). Comparison of Italian and Australian data showed that absence of a yqiL amplicon is not associated with any specific ST or CC. Although isolates that lack a target gene are not assigned an ST in the Strep. uberis PubMLST scheme, some MLST schemes do assign ST to such isolates, allowing for their inclusion in subsequent analyses and monitoring of their dissemination
(Zadoks et al., 2005; Pérez-Trallero et al., 2007). In a study of Streptococcus pyogenes isolates from Spain, the yqiL-negative ST403 was, in fact, the most prevalent clone detected. Inclusion of yqiL-deficient Strep. uberis isolates in the PubMLST scheme could be considered. Alternatively, genes that can be amplified from every isolate (e.g., gapC) could be used to replace yqiL in an MLST scheme. Although alteration of an MLST scheme would be labor intensive, precedent exists and modification of MLST schemes may result in enhanced resolution (Thomas et al., 2007) or improved understanding of the population structure of a pathogen (Sørensen et al., 2010).

\section{CONCLUSIONS}

Ovine and bovine mastitis appear to be caused by distinct subpopulations of Strep. uberis, with regard to both housekeeping and virulence genes. Because of the highly recombinatorial nature of Strep. uberis, lateral gene transfer may result in exchange of genetic material between bacterial subpopulations associated with different host species and between streptococcal species. This could affect the efficacy of Strep. uberis mastitis vaccines, for example, if the composition of the ovine Strep. uberis population is not considered during vaccine development and evaluation.

\section{ACKNOWLEDGMENTS}

We thank Francesco Cancellotti (Istituto Zooprofilattico Sperimentale delle Regioni Lazio e Toscana, Rome, Italy) for the provision of ovine and bovine Strep. uberis isolates and James Leigh (School of Veterinary Medicine and Science, The University of Nottingham, UK) for advice on detection and characterization of plasminogen activator genes. This study was financially supported by Pfizer Animal Health (New York, NY). The publication made use of the Strep. uberis MLST website (http://pubmlst.org/suberis) developed by Keith Jolley and located at the University of Oxford (Jolley et al., 2004).

\section{REFERENCES}

Ben Zakour, N. L., C. M. Guinane, and J. R. Fitzgerald. 2008. Pathogenomics of the staphylococci: Insights into niche adaptation and the emergence of new virulent strains. FEMS Microbiol. Lett. 289:1-12.

Bradley, A. J., K. A. Leach, J. E. Breen, L. E. Green, and M. J. Green. 2007. Survey of the incidence and aetiology of mastitis on dairy farms in England and Wales. Vet. Rec. 160:253-257.

Coffey, T. J., G. D. Pullinger, R. Urwin, K. A. Jolley, S. M. Wilson, M. C. Maiden, and J. A. Leigh. 2006. First insights into the evolution of Streptococcus uberis: A multilocus sequence typing scheme that enables investigation of its population biology. Appl. Environ. Microbiol. 72:1420-1428. 
Cuccuru, C., M. Meloni, E. Sala, L. Scaccabarozzi, C. Locatelli, P. Moroni, and V. Bronzo. 2011. Effects of intramammary infections on somatic cell score and milk yield in Sarda sheep. N. Z. Vet. J. 59:128-131.

Fontaine, M. C., J. Perez-Casal, X. M. Song, J. Shelford, P. J. Willson, and A. A. Potter. 2002. Immunisation of dairy cattle with recombinant Streptococcus uberis GapC or a chimeric CAMP antigen confers protection against heterologous bacterial challenge. Vaccine 20:2278-2286.

Gülcü, H. B., and H. Öngör. 2002. Bacteriological examination of the udder samples collected from sheep and goats slaughtered at a local abattoir in Elazig. Veteriner Bilimleri Dersigi 18:67-69.

Hariharan, H., W. Donachie, C. Macaldowie, and G. Keefe. 2004. Bacteriology and somatic cell counts in milk samples from ewes on a Scottish farm. Can. J. Vet. Res. 68:188-192.

Hassan, A. A., I. U. Khan, A. Abdulmawjood, and C. Lämmler. 2001. Evaluation of PCR methods for rapid identification and differentiation of Streptococcus uberis and Streptococcus parauberis. J. Clin. Microbiol. 39:1618-1621.

Jolley, K. A., M. S. Chan, and M. C. Maiden. 2004. mlstdbNet-Distributed multi-locus sequence typing (MLST) databases. BMC Bioinformatics 5:86.

Jones, M. N., and R. G. Holt. 2004. Activation of plasminogen by Streptococcus mutans. Biochem. Biophys. Res. Commun. 322:3741.

Khan, I. U., A. A. Hassan, A. Abdulmawjood, C. Lämmler, W. Wolter, and M. Zschöck. 2003. Identification and epidemiological characterization of Streptococcus uberis isolated from bovine mastitis using conventional and molecular methods. J. Vet. Sci. 4:213-224.

Kirk, J. H., E. M. Huffman, and B. C. Anderson. 1980. Mastitis and udder abnormalities as related to neonatal lamb mortality in shedlambed range ewes. J. Anim. Sci. 50:610-616.

Kitt, A. J., and J. A. Leigh. 1997. The auxotrophic nature of Streptococcus uberis. The acquisition of essential acids from plasmin derived casein peptides. Adv. Exp. Med. Biol. 418:647-650.

Lang, P., T. Lefébure, W. Wang, R. N. Zadoks, Y. H. Schukken, and M. J. Stanhope. 2009. Gene content differences across strains of Streptococcus uberis identified using oligonucleotide microarray comparative genomic hybridization. Infect. Genet. Evol. 9:179 188

Leigh, J. A., J. M. Finch, T. R. Field, N. C. Real, A. Winter, A. W. Walton, and S. M. Hodgkinson. 1999. Vaccination with the plasminogen activator from Streptococcus uberis induces an inhibitory response and protects against experimental infection in the dairy cow. Vaccine 17:851-857.

López-Benavides, M. G., J. H. Williamson, G. D. Pullinger, S. J. LacyHulbert, R. T. Cursons, and J. A. Leigh. 2007. Field observations on the variation of Streptococcus uberis populations in a pasturebased dairy farm. J. Dairy Sci. 90:5558-5566.

McDougall, S., T. J. Parkinson, M. Leyland, F. M. Anniss, and S. G. Fenwick. 2004. Duration of infection and strain variation in Streptococcus uberis isolated from cows' milk. J. Dairy Sci. 87:20622072.

Nam, H. M., S. Lim, H. Kang, J. Kim, J. Moon, K. Jang, Y. Joo, M. Kang, and S. Jung. 2009. Antimicrobial resistance of streptococci isolated from mastitic bovine milk samples in Korea. J. Vet. Diagn. Invest. 21:698-701.

Olde Riekerink, R. G., H. W. Barkema, D. F. Kelton, and D. T. Scholl. 2008. Incidence rate of clinical mastitis on Canadian dairy farms. J. Dairy Sci. 91:1366-1377.

Pérez-Trallero, E., M. Montes, B. Orden, E. Tamayo, J. M. GarcíaArenzana, and J. M. Marimón. 2007. Phenotypic and genotypic characterization of Streptococcus pyogenes isolates displaying the MLSB phenotype of macrolide resistance in Spain, 1999 to 2005. Antimicrob. Agents Chemother. 51:1228-1233.

Pullinger, G. D., T. J. Coffey, M. C. Maiden, and J. A. Leigh. 2007. Multilocus-sequence typing analysis reveals similar populations of Streptococcus uberis are responsible for bovine intramammary infections of short and long duration. Vet. Microbiol. 119:194-204.
Pullinger, G. D., M. López-Benavides, T. J. Coffey, J. H. Williamson R. T. Cursons, E. Summers, J. Lacy-Hulbert, M. C. Maiden, and J. A. Leigh. 2006. Application of Streptococcus uberis multilocus sequence typing: Analysis of the population structure detected among environmental and bovine isolates from New Zealand and the United Kingdom. Appl. Environ. Microbiol. 72:1429-1436.

Rato, M. G., R. Bexiga, S. F. Nunes, L. M. Cavaco, C. L. Vilela, and I. Santos-Sanches. 2008. Molecular epidemiology and population structure of bovine Streptococcus uberis. J. Dairy Sci. 91:45424551

Richards, V. P., P. Lang, P. D. Bitar, T. Lefébure, Y. H. Schukken, R. N. Zadoks, and M. J. Stanhope. 2011. Comparative genomics and the role of lateral gene transfer in the evolution of bovine adapted Streptococcus agalactiae. Infect. Genet. Evol. 11:1263-1275.

Rossitto, P. V., L. Ruiz, Y. Kikuchi, K. Glenn, K. Luiz, J. L. Watts, and J. S. Cullor. 2002. Antibiotic susceptibility patterns for environmental streptococci isolated from bovine mastitis in central California dairies. J. Dairy Sci. 85:132-138.

Sampimon, O., H. W. Barkema, I. Berends, J. Sol, and T. Lam. 2009. Prevalence of intramammary infection in Dutch dairy herds. J. Dairy Res. 76:129-136.

Shum, L. W., C. S. McConnel, A. A. Gunn, and J. K. House. 2009 Environmental mastitis in intensive high-producing dairy herds in New South Wales. Aust. Vet. J. 87:469-475.

Sørensen, U. B., K. Poulsen, C. Ghezzo, I. Margarit, and M. Kilian. 2010. Emergence and global dissemination of host-specific Streptococcus agalactiae clones. MBio 1:e00178-10.

Sun, H., U. Ringdahl, J. W. Homeister, W. P. Fay, N. C. Engleberg, A. Y. Yang, L. S. Rozek, X. Wang, U. Sjöbring, and D. Ginsburg. 2004. Plasminogen is a critical host pathogenicity factor for group A streptococcal infection. Science 305:1283-1286.

Thomas, J. C., M. R. Vargas, M. Miragaia, S. J. Peacock, G. L. Archer, and M. C. Enright. 2007. Improved multilocus sequence typing scheme for Staphylococcus epidermidis. J. Clin. Microbiol. 45:616-619.

Tomita, T., B. Meehan, N. Wongkattiya, J. Malmo, G. Pullinger, L. Leigh, and M. Deighton. 2008. Identification of Streptococcus uberis multilocus sequence types highly associated with mastitis. Appl. Environ. Microbiol. 74:114-124.

Vautor, E., V. Magnone, G. Rios, K. Le Brigand, D. Bergonier, G Lina, H. Meugnier, P. Barbry, R. Thiéry, and M. Pépin. 2009. Genetic differences among Staphylococcus aureus isolates from dairy ruminant species: A single-dye DNA microarray approach. Vet. Microbiol. 133:105-114.

Ward, P. N., and J. A. Leigh. 2002. Characterization of PauB, a novel broad-spectrum plasminogen activator from Streptococcus uberis. J. Bacteriol. 184:119-125.

Ward, P. N., M. T. Holden, J. A. Leigh, N. Lennard, A. Bignell, A. Barron, L. Clark, M. A. Quail, J. Woodward, B. G. Barrell, S A. Egan, T. R. Field, D. Maskell, M. Kehoe, C. G. Dowson, N. Chanter, A. M. Whatmore, S. D. Benley, and J. Parkhill. 2009. Evidence for niche adaptation in the genome of the bovine pathogen Streptococcus uberis. BMC Genomics 10:54.

Zadoks, R. N., R. González, K. J. Boor, and Y. H. Schukken. 2004. Mastitis-causing streptococci are important contributors to bacterial counts in raw bulk tank milk. J. Food Prot. 67:2644-2650.

Zadoks, R. N. 2007. Sources and epidemiology of Streptococcus uberis, with special emphasis on mastitis in dairy cattle. CAB Reviews: Perspectives in Agriculture, Veterinary Science, Nutrition and Natural Resources 2(030):15.

Zadoks, R. N., J. R. Middleton, S. McDougall, J. Katholm, and Y. H. Schukken. 2011. Molecular epidemiology of mastitis pathogens of dairy cattle and comparative relevance to humans. J. Mammary Gland Biol. Neoplasia 16:357-372.

Zadoks, R. N., Y. H. Schukken, and M. Wiedmann. 2005. Multilocus sequence typing of Streptococcus uberis provides sensitive and epidemiologically relevant subtype information and reveals positive selection in the virulence gene pauA. J. Clin. Microbiol. 43:24072417 